\title{
Interactive comment on "Towards a webcam-based snow cover monitoring network: methodology and evaluation" by Céline Portenier et al.
}

Céline Portenier et al.

celine.portenier@giub.unibe.ch

Received and published: 28 September 2019

We thank A. N. Arslan for his valuable and constructive comments that were helpful to improve the manuscript.

Below we respond to all comments by A. N. Arslan. The responses (normal font style) are following the referees' comments (displayed in italic font style) directly.

\section{General comments:}

The tc-2019-142 manuscript, entitled," Towards a webcam-based snow cover monitoring network: methodology and evaluation" presents a semi-automatic approach proce- 
dure to derive snow cover maps. The semi-automatic approach procedure is consist of (1) automatic image to image alignment and (2) automatic image to DEM registration which are the contributions of the manuscript. In addition a snow classification method (two existing methods in literature presented) and a manual user input (for estimation webcam's location) are needed for estimating snow cover from a webcam image.

The purpose of the work is clearly articulated and the methodology and results are adequately presented.

Specific Comments:

There are following issues which I believe need more discussions such as

(1) webcam-based snow cover monitoring network

(2) Arbitrary images to generate snow cover maps

(3) Most of existing studies use single cameras and thus are limited in areal coverage. In particular, they either require known camera parameters (i.e., extrinsic and intrinsic camera parameters such as the camera orientation or the FOV of the camera) or require significant manual user input (e.g., ground control points (GCPs)) to georectify terrestrial photography

(4) Since camera parameters are not readily available for public webcams, and manually setting GCPs for a large number of cameras is time-consuming, these methods are of limited application for our purposes.

(1) webcam-based snow cover monitoring: This is very good concept. It is very good to explain this concept in more detail and how the proposed methodology can be applied and what current status of existing webcam networks is. What should be done apart from improving snow classification methods mentioned in the manuscript?

Thank you for pointing this out. We agree to add more details about the possible applications of using webcam images for snow cover monitoring. We will extend the discussion on possible applications and improvements of our methodology.

Printer-friendly version

(2) I am not sure about arbitrary images as one should know the location of camera. 
We agree that this is a bit overstated. We will remove the word 'arbitrary' in the revision of the manuscript, and mention that the location must be estimateable.

(3) It is not clear for me what differences are! In the proposed procedure in the manuscript one has to create "master image" How more easy and accurate is creating master image than procedures in the existing studies?

Selecting a single Master image per webcam is straightforward, the only assumption is that the daytime and weather conditions are such that the mountain silhouette is clearly visible. In contrast, having access to intrinsic and extrinsic camera parameters, or measuring these parameters using GCPs is infeasible for a reasonably large-scale camera network. Since our method computes these parameters using only the Master image and camera position estimation as input, it is feasible to compile a large-scale camera network with our approach. However, it is true that the accuracy of our imageto-DEM mapping is expected to be lower compared to approaches where ground truth camera parameters are available. We will discuss this in the revised text.

(4) How about camera locations? How do one get locations of webcams which are need as input in the proposed manuscript? As the objective is "towards webcam-based snow cover monitoring" why not setting GCPs for time-consuming. The creating an accurate master image is an essential part of the proposed work in the manuscript.How time consuming is creating a good master image? What is applicability of creating master image in various environment as silhouette extraction is based on the assumption that the mountain silhouette in the manuscript. How about open and forested areas isn't it big limitations of the method towards webcam-based snow cover monitoring network? That's why all should be explained!

Printer-friendly version

We manually estimate webcam locations by considering the position of objects visible in the webcam image, the orthophoto SWISSIMAGE, and additional information provided by the webcam owner. This can be, for example, the name of a restaurant 
(Section 3.1). It is correct that webcams cannot be used by our approach if they do not feature mountain silhouettes due to open or forested areas, or where the silhouette is partially occluded by trees or buildings. We will discuss this in the revised version of the manuscript.

There is an evaluation on the accuracy of the automatic image-to-DEM registration. There is no an evaluation of the proposed procedure, entitled, "a semi-automatic approach procedure".

We agree that the main focus of our evaluation lies on the automatic image-to-DEM registration, which we consider our main contribution. We did not explicitly evaluate parts of our pipeline that we adopted. However, we provide a qualitative comparison of the leveraged snow classification techniques.

As it is mentioned at the end of the discussion in the manuscript "our webcam snow cover maps facilitate the gap filling of partly cloud-obscured satellite-based snow cover maps or improve snow classification in steep terrain or shadow-affected image scenes." It would be good to see some evaluation of the proposed procedure supporting this statement.

We agree that it is an important further step to apply our proposed framework to perform such evaluations. However, this would be out of scope for the current work and we consider this as future work. We will mention this in the discussion section.

Technical Corrections:

In Figure 11: It is good to explain colors like red and blue; which one is Salvotori et.al method and etc.

Thank you for pointing this out. We will describe the color coding in the figure caption.

Interactive comment on The Cryosphere Discuss., https://doi.org/10.5194/tc-2019-142, 2019. 